\title{
The Effect of Parasites on Host Population Density and Extinction: Experimental Epidemiology with Daphnia and Six Microparasites
}

\section{Citation}

Ebert, Dieter, Marc Lipsitch, and Katrina L. Mangin. 2000. “The Effect of Parasites on Host Population Density and Extinction: Experimental Epidemiology with Daphnia and Six Microparasites." The American Naturalist 156 (5): 459-77. https://doi.org/10.1086/303404.

\section{Permanent link}

http://nrs.harvard.edu/urn-3:HUL.InstRepos:41426759

\section{Terms of Use}

This article was downloaded from Harvard University's DASH repository, and is made available under the terms and conditions applicable to Other Posted Material, as set forth at http:// nrs.harvard.edu/urn-3:HUL.InstRepos:dash.current.terms-of-use\#LAA

\section{Share Your Story}

The Harvard community has made this article openly available.

Please share how this access benefits you. Submit a story.

Accessibility 


\title{
The Effect of Parasites on Host Population Density and Extinction: Experimental Epidemiology with Daphnia and Six Microparasites
}

\author{
Dieter Ebert, ${ }^{1,2,3, *}$ Marc Lipsitch, ${ }^{4}$ and Katrina L. Mangin ${ }^{1, \dagger}$
}

1. Department of Zoology, Oxford University, South Parks Road, Oxford OX1 3PS, United Kingdom;

2. Natural Environment Research Council Center for Population Biology, Imperial College at Silwood Park, Ascot, Berkshire SL5

7PY, United Kingdom;

3. Basel University, Institut für Zoologie, Rheinsprung 9, CH-4051 Basel, Switzerland;

4. Department of Epidemiology, Harvard School of Public Health, Boston, Massachusetts 02115

Submitted May 25, 1999; Accepted June 30, 2000

ABstract: Parasites have been shown to reduce host density and to induce host population extinction in some cases but not in others. Epidemiological models suggest that variable effects of parasites on individual hosts can explain this variability on the population level. Here, we aim to support this hypothesis with a specific epidemiological model using a cross-parasite species approach. We compared the effect of six parasites on host fecundity and survival to their effects on density and risk of extinction of clonal host populations. We contrast our empirical results of population density with predictions from a deterministic model and contrast our empirical results of host and parasite extinction rates with those predicted by a stochastic model. Five horizontally transmitted microparasites (two bacteria: white bacterial disease, Pasteuria ramosa; two microsporidia: Glugoides intestinalis, Ordospora colligata; one fungus: Metschnikowiella biscuspidata); and six strains of a vertically transmitted microsporidium (Flabelliforma magnivora) of the planktonic crustacean Daphnia magna were used. In life table experiments, we quantified fecundity and survival in individual parasitized and healthy hosts and compared these with the effect of the parasites on host population density and on the likelihood of host population extinction in microcosm populations. Parasite species varied strongly in their effects on host fecundity, host survival, host density reduction, and

\footnotetext{
* Author to whom correspondence should be addressed. Present address: Basel University, Institut für Zoologie, Rheinsprung 9, CH-4051 Basel, Switzerland; e-mail: d.ebert@unibas.ch.

${ }^{\dagger}$ Present address: University of Arizona, Department of Ecology and Evolutionary Biology, Tucson, Arizona 85721.
}

Am. Nat. 2000. Vol. 156, pp. 459-477. (c) 2000 by The University of Chicago. 0003-0147/2000/15605-0001\$03.00. All rights reserved. the frequency with which they drove host populations to extinction. The fewer offspring an infected host produced, the lower the density of an infected host population. This effect on host density was relatively stronger for the vertically transmitted parasite strains than for the horizontally transmitted parasites. As predicted by the stochastic simulations, strong effects of a parasite on individual host survival and fecundity increased the risk of host population extinction. The same was true for parasite extinctions. Our results have implications for the use of microparasites in biological control programs and for the role parasites play in driving small populations to extinction.

Keywords: experimental epidemiology, microparasites, Daphnia magna, host regulation, host extinction, individual and population level effects.

Experimental and observational studies have shown that parasites can reduce host density and even drive host populations to extinction (Park 1948; Finlayson 1949; Keymer 1981; Dobson and Hudson 1986; Scott and Dobson 1989; Minchella and Scott 1991; Hudson et al. 1992, 1998; Kohler and Wiley 1992; Roelke-Parker et al. 1996). These studies provide strong evidence that parasites are able to regulate their host populations and that some parasites are more likely to do so than others. What is now needed to make empirical work in epidemiology more predictive are experiments that demonstrate a correlation between the effects of parasites on individual hosts and the ability of a parasite to regulate host population density. Theory on the effects of parasites on host population dynamics has been extensively developed, with the aim of understanding whether variability in the effects of parasites on host fecundity and survival are reflected in host population dynamics (May and Anderson 1978, 1979, 1983; Anderson and May 1979, 1981, 1986, 1991; reviewed by McCallum 1995; McCallum and Dobson 1995). A key question is whether processes at one level (individual) translate into effects at another level (population). For a number of hostparasite systems, we have good empirical data on individual-level processes (e.g., pathogenicity) but little on pop- 
ulation-level processes. The aim of this study was to test whether the extent to which a parasite reduces the fecundity and survival of individual infected hosts can be used to predict the ability of a parasite to regulate its host population. We are particularly interested in microparasites, that is, small, unicellular parasites that have direct reproduction within their hosts (Anderson and May 1979).

Mathematical models predict different population dynamics for hosts infected with microparasites that reduce host fecundity versus parasites that reduce host survival (Anderson 1979, 1982). Host density is predicted to decrease monotonically with the negative effect that a parasite has on host fecundity (all other things being equal). In contrast, if parasites reduce host survival, then mean host population density is predicted to first decrease and then increase with an increase in parasite-induced host mortality. This is because (for a given transmission rate parameter) parasites that kill their hosts very rapidly are less likely to be transmitted to other hosts and therefore will remain at low prevalence, while parasites with little effect on host mortality will have little effect on host demographics. A further prediction made by these epidemiological models concerns population fluctuations. Host density fluctuations are predicted to increase with an increasing negative effect of a microparasite on host survival and fecundity. Therefore, when host populations are small, density fluctuations are expected to increase the chance of host extinction because host density is more likely to drop to 0 during population bottlenecks (May 1974; McCallum and Dobson 1995).

Epidemiological models, such as those cited above, are often used to explain empirical results where parasites reduce the density or lead to the extinction of their host populations. The same models predict little effect of benign parasites on host population densities and so can apply equally well to cases where parasites have little or no apparent effect on host population dynamics. Therefore, besides contrasting parasitized with nonparasitized populations, it is important to compare host populations infected by parasites with different effects on host fecundity and survival. We used five species of horizontally transmitted microparasites that varied in their effects on their host, the planktonic crustacean Daphnia magna, to test whether the predicted relationship between individual effects and population effects hold across parasites.

To provide a conceptual framework for our study, we formulate a simple mathematical model of a horizontally transmitted microparasite that affects the fecundity and mortality of its host, which in turn is regulated both by density-dependent birth and the parasite. The model is based on earlier models (Anderson 1979, 1982) but is specifically tailored to the microparasite-Daphnia system. Because the model is deterministic, it does not allow us to predict host and parasite extinctions. Therefore, we use a stochastic version of the model to simulate extinction probabilities.

We contrast the results of the five species of horizontally transmitted microparasites with similar data from six strains of one species of a vertically transmitted microsporidium. This parasite is vertically transmitted with nearly $100 \%$ efficiency and reduces host fecundity by 30\%-60\% (Mangin et al. 1995). Under natural conditions, a harmful parasite with exclusively vertical transmission cannot exist (Fine 1975; Bull et al. 1991; Herre 1993; Lipsitch et al. 1995; Mangin et al. 1995) because competition with uninfected hosts will drive it to extinction. The vertically transmitted parasite species used in our experiments was maintained in our laboratory cultures, even in the absence of horizontal transmission, by excluding competition with uninfected hosts. In nature, a harmful and vertically transmitted parasite can be maintained only with an additional mechanism for horizontal transmission, which is the likely explanation for the persistence of this parasite (Mangin et al. 1995).

\section{The Host-Parasite System and a Mathematical Model}

The Daphia magna-microparasite system is particularly suitable for experimental epidemiology because it can be well described with simple mathematical models. The relevant points of the biology of the system follow. First, population growth of D. magna in laboratory populations with a constant food supply and no parasites is reasonably well described by a logistic-growth model. In the absence of parasites, Daphnia populations reach an equilibrium population level, representing the carrying capacity (e.g., Perrin et al. 1992). Second, the Daphnia parasites used here fit well the definition used for epidemiological models of microparasites as small, unicellular parasites that have direct reproduction within their hosts and are directly transmitted (Anderson and May 1979). Third, all microparasites used in our study produced persistent infections without host recovery; therefore, only two classes of hosts need to be considered in the model-infected and uninfected hosts. Fourth, under laboratory conditions $\left(20^{\circ} \mathrm{C}\right)$, D. magna produce their first eggs after $7-15 \mathrm{~d}$ (depending on food level), followed by a clutch of parthenogenetic eggs every 3-4 d until death, resulting in an approximately constant fecundity across the adult life of $D$. magna. The first clutch is usually smaller than subsequent clutches. Generations overlap. Intraspecific competition for food decreases fecundity, and severe food shortages may result in skipped clutches or delayed maturity. Fifth, controlled laboratory conditions allow other extrinsic sources of mortality (besides infection by parasites) to be excluded. Sixth, differential equation models of the type discussed here use 

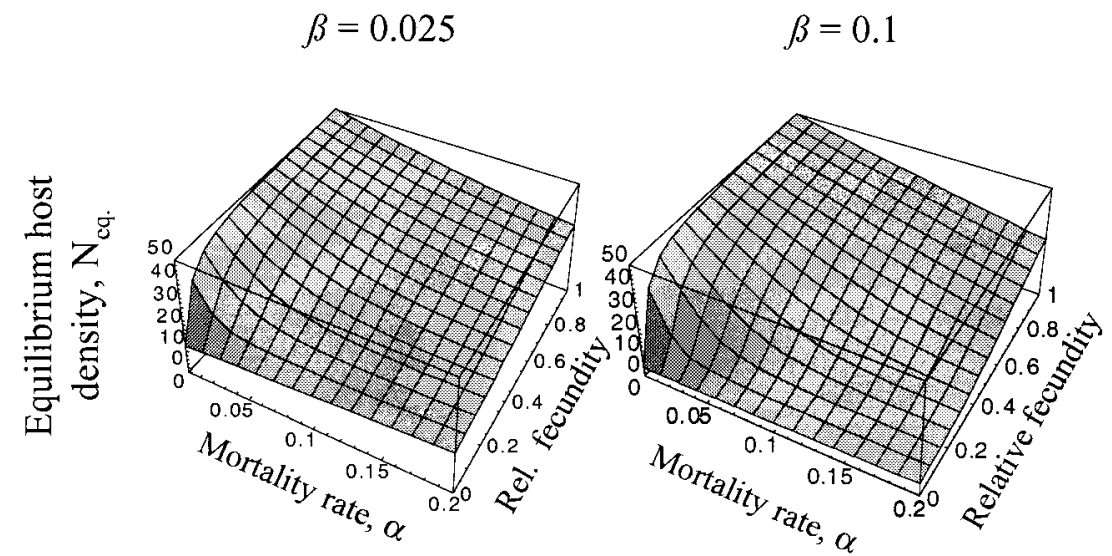

Figure 1: Equilibrium density $\left(N_{e q}\right)$ of host populations for our microcosm experiments as predicted by the mathematical model. Parameter values: $d=0.01 / \mathrm{d}$ (life span in the absence of parasite $>50 \mathrm{~d}$ ), $a=0.4 / \mathrm{d}$ (maximum birth rate in density-free conditions), $\alpha=0$ to $0.2 / \mathrm{d}$ ( $1 /$ time to death), and $\beta=0.025 / \mathrm{d}$ and $0.1 / \mathrm{d}$.

birth and death rates that are unconstrained by density dependence, that is, rates that reflect host growth in the absence of intraspecific competition. Such rates can be easily obtained for infected and uninfected D. magna, by using life table experiments with individually cultured host females. Seventh, simple epidemiological models usually assume homogeneously mixed populations without spatial and genetic structure. Monoclonal Daphnia populations in the laboratory fulfill these criteria. The latter point is a particularly advantageous aspect of this experimental system because the absence of genetic host diversity allows us to exclude the confounding effect of host evolution. In monoclonal Daphnia populations, no genetic diversity can arise, except by mutations. Eighth, clonally reproducing hosts allow us to avoid complications because of mate choice and mate finding. The clones from England used in our experiment produced few to no males during the experiments. The clones from Russia occasionally produced males but never in large numbers.

\section{A Mathematical Model}

We consider a model of microparasite transmission for a horizontally transmitted parasite that reduces fecundity and survival of its host, which in turn is regulated both by density-dependent birth and by the parasite-induced mortality. Although our model is tailored to the Daphniamicroparasite system, it is representative of a general class of epidemiological microparasite models. The model is nearly identical to that of McCallum (1994), which is based on models by Anderson (1979) and Anderson and May (1979). We have modified McCallum's model by assuming density-dependent birth (rather than death) and by ig- noring the possibility of host recovery. The model equations are

$$
\begin{aligned}
& d x / d t=a(x+f y)[1-c(x+y)]-d y-\beta x y, \\
& d y / d t=\beta x y-(d+\alpha) y
\end{aligned}
$$

where $x$ and $y$ are the densities of uninfected and infected hosts (per $100 \mathrm{~mL}$ medium), respectively; $a$ is the maximum per capita birth rate of uninfected hosts; $f$ is the relative fecundity of an infected host; $c$ measures the per capita density-dependent reduction in birth rate; $d$ is the parasite-independent host background mortality; $\beta$ is the infection rate constant; and $\alpha$ is the parasite-induced excess death rate.

This model predicts the existence of a stable equilibrium of infected and uninfected hosts, and the population is predicted to approach this equilibrium either monotonically or by damped oscillations. It further predicts that equilibrium host density will decrease monotonically as a function of parasite-induced reductions in fecundity (fig. 1). This monotonic decline is a general feature of our and similar models (e.g., Anderson 1979; Anderson and May 1979; McCallum 1994). In contrast to the effect of the parasite on host fecundity, the relationship between equilibrium host density and parasite effect on mortality in our model, as in McCallum's (1994) model, can be monotonically increasing, monotonically decreasing, or reach a minimum for intermediate mortality effects (see also McCallum and Dobson 1995 for a graphical presentation of a similar model). However, for most parameter values that are reasonable for the Daphnia-microparasite system $(d<0.02 / \mathrm{d}$ [life span in the absence of parasite $>50 \mathrm{~d}$ ], $a=0.4 / \mathrm{d}$ [maximum birth rate in the absence of com- 
petition], $\alpha=0-0.2 / \mathrm{d}$ [disease induced excess mortality rate], $\beta=0.01$ to $0.1 / \mathrm{d} /$ host), the equilibrium population size is almost always a decreasing function of the parasiteinduced mortality. The only exceptions are for low levels of $f$ and $\beta$ and for high levels of $\alpha$ (fig. 1).

\section{Stochastic Simulation of Host and Parasite Extinction}

To model the effect of parasite-induced mortality and reduced fecundity on host population viability, we performed simulations of a stochastic model corresponding to the deterministic model described above. To do so, we simulated a continuous-time Markov process in which four events were possible: birth of an uninfected host, death of an uninfected host, death of an infected host, and infection of a previously uninfected host. Instantaneous rate parameters for these events were given by the corresponding terms in the deterministic model. Initially, we performed simulations for which the state space was defined by a pair of integers $(x, y)$ representing numbers of uninfected and infected hosts, respectively. These proved unsatisfactory, however, because such a simple model in which the per capita mortality rate is constant imposes exponentially distributed life spans on the hosts (conditional on no change in their infection status). As will become evident in the "Results" section, the life spans of infected hosts are not exponentially distributed, and this resulted in poor results from the simulations (data not shown). A better fit to the sigmoid survivorship data was obtained by using Weibull distributions (Klein and Moeschberger 1997).

We therefore increased the complexity of the simulations to include an age-time since-infection structure. In these simulations, we kept track of the number of uninfected hosts of each age ( $\mathrm{d}$ since birth) and the number of infected hosts based on their time since infection. Thus, the state space was composed of pairs of vectors $(\boldsymbol{X}, \boldsymbol{Y})$, where $\boldsymbol{X}^{T}=\left(x_{0}, x_{1}, x_{2}, \ldots x_{m}\right)$ and $\boldsymbol{Y}^{T}=$ $\left(y_{0}, y_{1}, y_{2}, \ldots y_{m}\right)$, where $x_{i}$ is the (integer) number of uninfected Daphnia born $i \mathrm{~d}$ ago and $y_{i}$ is the (integer) number of infected Daphnia that were infected $i \mathrm{~d}$ ago. The total number of uninfected and infected hosts are given, respectively, by $X$ and $Y$. The transitions in this structured model occur as shown in table 1 . Here, $u_{i}$ is the death rate per day of a host infected $i \mathrm{~d}$ ago, and $d_{i}$ is the death rate per day of an uninfected host aged $i \mathrm{~d}$. Survivorship for the hypothetical parasites in these simulations was modeled by Weibull distributions with 5-, 10-, 20-, 40-, and 80-d median survival times and a slope such that $95 \%$ of the parasitized hosts were dead $10 \mathrm{~d}$ after the median survival time. A Weibull hazard function is given by $h(t)=\lambda \alpha t^{\alpha-1}$, and parameters for a median survival time of $T \mathrm{~d}$ and $95 \%$ mortality by $T+10 \mathrm{~d}$ are given by $\alpha=\ln [(\ln 0.05) /(\ln 0.5)] / \ln [(T+10) / T)], \lambda=$ $-\ln 0.5 / T^{\alpha}$ (Klein and Moeschberger 1997). We assumed that mortality in infected hosts depended only on their time since infection, not on their age. Other parameters were as described above for the deterministic simulations.

We performed stochastic simulations with parameter values chosen to span the range of plausible parasiteinduced mortality and fecundity reductions for the parasites of Daphnia. This was done to investigate systematically the effects of these forms of parasite-induced reductions of host fitness on the extinction probabilities of host and parasite populations. Simulations were performed in FORTRAN (program available on request) with fecundity reductions ranging from $0(f=1)$ to complete $(f=0)$ and median time to host death of 5, 10, 20, 40, and $80 \mathrm{~d}$. The simulations for the estimated parameters of the parasites were begun on day 0 with five infected and five uninfected hosts to mimic the experiments. Each simulated day, the vectors were updated to increase the age-time since infection of each individual by one.

The probability of extinction of a host population is a combined function of low relative fecundity, $f$, and parasite induced host death (fig. 2). With very low values of $f$, host populations go extinct in nearly all cases. However, with increasing $f$, the host extinction risk drops drastically, except when infected hosts have a short life expectancy because of infection (fig. 2). Host extinction risks increase when $\beta$ increases. The results for parasite extinction look very similar to those of host extinction. This is partially because the parasite goes extinct whenever the host goes extinct, but for low values of $f$ and median host survival time, the parasite goes extinct in many cases where the host population survives (fig. 2).

We did not perform simulations for the nearly $100 \%$ vertically transmitted parasite strains (Flabelliforma magnivora). Parasite extinction in these cases is expected to be very low and may occur only in the rare cases of non-

Table 1: Transitions used in the stochastic model

\begin{tabular}{llc}
\hline Transition & \multicolumn{1}{c}{ Change } & Instantaneous rate \\
\hline Birth of an uninfected host & $x_{0} \rightarrow x_{0}+1$ & $a(X+f Y)[1-c(X+Y)]$ \\
Death of an uninfected host aged $i \mathrm{~d}$ & $x_{i} \rightarrow x_{i}-1$ & $d_{i} x_{i}$ \\
Death of an infected host infected $i \mathrm{~d}$ ago & $y_{i} \rightarrow y_{i}-1$ & $u_{i} y_{i}$ \\
Infection of a host aged $i$ & $x_{i} \rightarrow x_{i}-1, y_{0} \rightarrow y_{0}+1$ & $\beta Y x_{i}$ \\
\hline
\end{tabular}


$\beta=0.025$

A

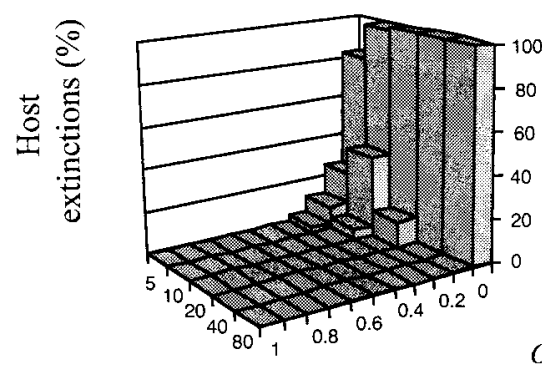

$\beta=0.1$

B
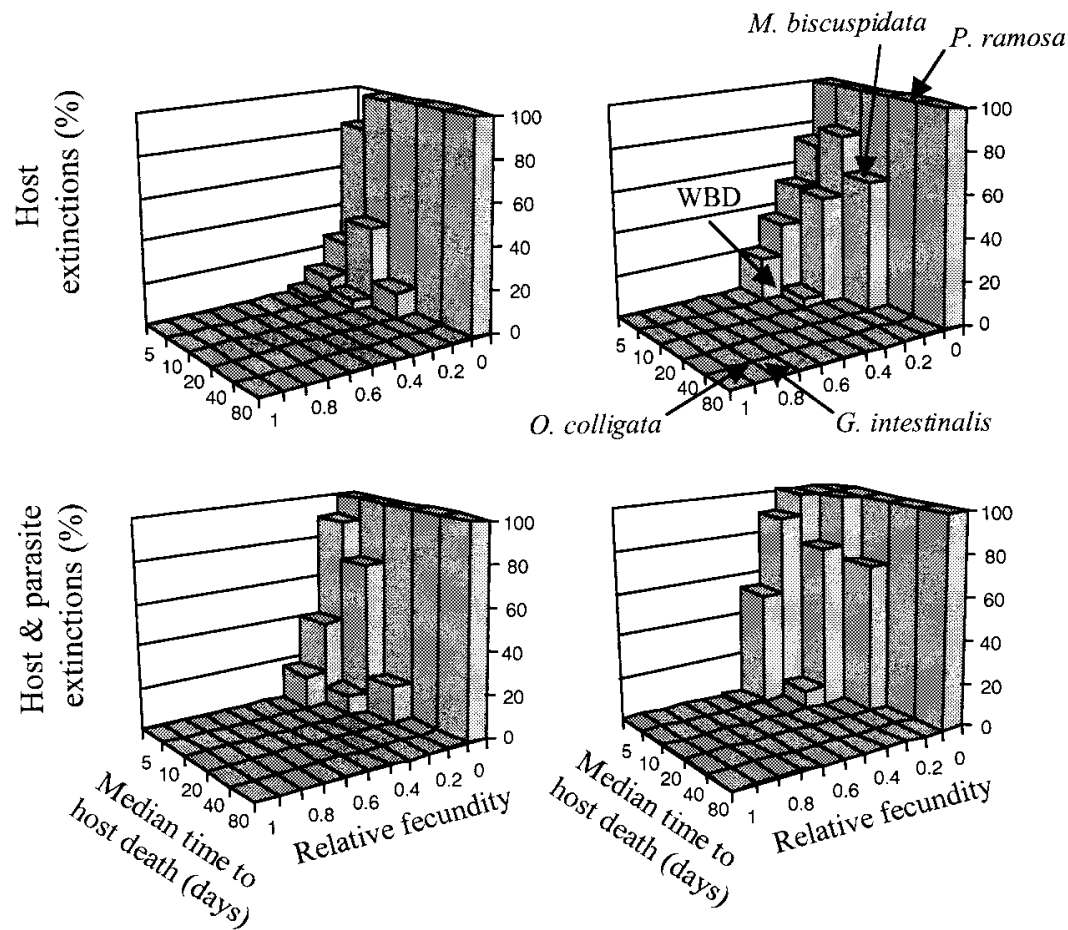

Figure 2: Proportion of host (upper graphs) and parasite (including host) extinctions (bottom graphs) in relation to relative fecundity, $f$, and the median time to host death, generated by stochastic simulation. For each combination of $f$, median survival time, and $\beta$, we performed 100 runs for $300 \mathrm{~d}$ with a population size of 50. The graphs show the proportion of runs in which extinctions occurred. In the upper left graph, we indicated the approximate position of the parasite-specific values for relative fecundity and time to host death. Note that the axis for median time to death is not evenly spaced. Note further, for better presentation of the graphs, the most benign parasites are located in the far corner of the graphs in figure 1 but in the very front of the graphs in figure 2.

perfect transmission, followed by competitive exclusion of the infected hosts by the healthy hosts. Host extinctions are also unlikely, as the parasites essentially behave like deleterious fixed genes in an asexual population. In the absence of competition and as long as the parasite does not reduce the host's $R_{0}$ below 1 , the host populations should not go extinct.

\section{Material and Methods}

We used Daphnia magna Straus (Crustacea: Cladocera) as the host in all experiments. Daphnia can be cloned easily and maintained asexually for many generations. Asexual generation time is $10-15 \mathrm{~d}$, although life span can be much longer. Daphnia clones used in this study were either bred from resting eggs (the Russian clone infected with the Flabelliforma parasite) or were isolated from Daphnia pop- ulations in Oxfordshire, United Kingdom (see Ebert 1994a, 1995). All experiments were done with host clones and parasite strain combinations derived from the same natural populations. We used six species of microparasites in our experiments (see table 1): one isolate each of two bacteria (Pasteuria ramosa and white bacterial disease [WBD]), one ascomycete fungus (Metschnikowiella biscuspidata), two microsporidia (Glugoides intestinalis and Ordospora colligata), and six isolates of a third species of microsporidium (Flabelliforma magnivora).

\section{Isolation and Maintenance of Parasites}

Parasites were isolated as described in Ebert (1994a). In short, infected females were collected from natural populations and kept singly under good food conditions in $100 \mathrm{~mL}$ of medium (about $10^{6}$ cell algae/d, 16L : 8D cycle, 
$20^{\circ} \mathrm{C}$ ). If the procedure was successful, the offspring of these females became infected through waterborne spores released from the dead or live mother. In cases where the parasite destroyed the ovaries of a female before she could reproduce, we placed uninfected newborn from a laboratory clone from the same $D$. magna population in the medium in order to continue to propagate the disease in that host genotype. Table 1 describes the parasites used in this study.

We isolated a vertically transmitted parasite from two sources. The Russian D. magna clone, which was infected with F. magnivora (Larsson et al. 1998), was hatched from a resting egg (=ephippium) from a pond $50 \mathrm{~km}$ north of Moscow. In an earlier publication, this parasite was referred to as Tuzetia sp. (Mangin et al. 1995). All offspring of this isofemale line were infected with the parasite. Five females infected with the same parasite were isolated from a pond south of Oxford, United Kingdom.

Since F. magnivora cannot be transmitted horizontally in the laboratory and vertical transmission is usually perfect (100\% to offspring), it was difficult to obtain a control of uninfected hosts from the same clone for each parasitehost combination. We succeeded in getting an uninfected host clone from the Russian clone (see Mangin et al. 1995) and from one of the five infected English D. magna clones. We used the single uninfected English clone as a control for all five infected isofemale lines in the population experiments (in the absence of true uninfected controls from the remaining four isofemale lines). However, during the following year, we managed to obtain the proper control clones for three of the remaining four other strains. These were used in the life table experiments. One of these controls served as a control for the fifth strain. Since genetic differences among the control clones in life-history traits were small (data not shown here) compared to the strong effects caused by the infections, we believe that this procedure does not bias our data.

Parasites were maintained in stock cultures of the host clone from which they were isolated. In some cases, we added uninfected hosts from the same host clone to these stock cultures to prevent host extinction. Daphnia magna reproduced only asexually in all experimental and control cultures.

\section{Experiments}

We used artificial Daphnia culture medium (Klüttgen et al. 1994) for all experiments and used a suspension of Chlamydomonas reinhardii (strain S1D2) as the sole food. The life table experiments were done in incubators with $16 \mathrm{~L}: 8 \mathrm{D}$ period and $20^{\circ} \mathrm{C}$. The population experiments were done at the same photoperiod but at room temperature $\left(18^{\circ}-22^{\circ} \mathrm{C}\right)$.
Life Table Experiments. Life table experiments were designed to test for the impact of parasites on individual hosts. To do this, we used a split-brood design for the horizontally transmitted parasites. We kept host females under standard conditions (see above) with $10^{5}$ cells algae/ $\mathrm{d} / \mathrm{mL}$ in $100-\mathrm{mL}$ beakers. From a female's second or later clutch, we randomly chose two newborns and placed each in a separate $100-\mathrm{mL}$ jar. One newborn served as a control while the other was infected by using a suspension of parasite spores. Spore suspensions were produced by collecting dead infected hosts from infected mass cultures and keeping them at $4^{\circ} \mathrm{C}$ until further use. When we had about 50 cadavers ( 25 for WBD), we concentrated them in $5 \mathrm{~mL}$ of water and homogenized their tissue. Homogenates were then diluted to $50 \mathrm{~mL}$ (25-mL WBD), and at day 2, 3, and 4 after birth, $0.5-\mathrm{mL}$ spore suspension was added to the beakers with the experimental hosts. The same procedure was repeated with uninfected Daphnia cadavers and administered to the controls. Host sample size (number of pairs) was 40 for G. intestinalis, P. ramosa, and M. biscuspidata and 20 for WBD (we were unable to obtain enough WBD spores for 40 replicates). Experiments were terminated no sooner than $10 \mathrm{~d}$ after the last infected host had died.

The life table data for O. colligata were taken from Mangin et al. (1995). In this publication, O. colligata was called Pleistophora intestinalis (see Larsson et al. 1997). This experiment ended at $46 \mathrm{~d}$, when only two out of 10 infected females had died (Mangin et al. 1995). Therefore, the mean age at death for hosts infected with $O$. colligata is in reality $>46 \mathrm{~d}$ (table 2).

The effect of the vertically transmitted microsporidium was tested with a slightly different procedure because a split-brood design was impossible with a vertically transmitted parasite (Mangin et al. 1995). Infected and uninfected hosts from the same clone were kept singly for two generations in $100-\mathrm{mL}$ glass jars under standard conditions. To start the experiment, we used pairs of newborns born on the same day. Each newborn came from a different mother, one from the infected host clone and the other from the uninfected host clone. We started with 10 host pairs per parasite strain. Because of technical problems during the experiments with the five English F. magnivora, we lost some replicates, which resulted in an unbalanced design. All vertically transmitted parasites were tested in the same experiment, and the experiment was terminated $5 \mathrm{~d}$, after the last infected host had died.

All beakers were checked once a day for host survival and reproduction. Newborns were counted and removed. Culture medium was changed at day 5 after the first spores were administered and then after every molt of adult hosts (about every 3-4 d). For each host, we recorded survival and the number of offspring produced. All animals (in- 
Table 2: Parasites of Daphnia magna used in the experiments

\begin{tabular}{|c|c|c|c|c|}
\hline Parasite & Route of transmission & $\begin{array}{l}\text { Infected tissue } \\
\text { (reference) }\end{array}$ & $\begin{array}{l}\text { Sampling location, } \\
\text { date of isolation }\end{array}$ & $\begin{array}{c}\text { Mean/maximum } \\
\text { prevalence }^{\mathrm{a}}\end{array}$ \\
\hline $\begin{array}{l}\text { White bacterial disease } \\
\text { (a coccoid bacterium) }\end{array}$ & $\begin{array}{l}\text { Direct, horizontal; af- } \\
\text { ter the death of the } \\
\text { host; waterborne }\end{array}$ & $\begin{array}{r}\text { Infects fat body } \\
\text { (Green 1974) }\end{array}$ & $\begin{array}{l}\text { Pond south of Ox- } \\
\text { ford, U.K. (pond } 1 \\
\text { in Ebert 1994a, } \\
1994 b \text { ), November } \\
1993\end{array}$ & $7.34 \% / 32 \%$ \\
\hline $\begin{array}{l}\text { Pasteuria ramosa } \\
\text { (bacterium) }\end{array}$ & $\begin{array}{l}\text { Direct, horizontal; af- } \\
\text { ter the death of } \\
\text { host; water- and } \\
\text { sediment-borne }\end{array}$ & $\begin{array}{l}\text { Infects hemolymph } \\
\text { (Metchnikoff 1888; } \\
\text { Ebert et al. 1996) }\end{array}$ & $\begin{array}{l}\text { Pond south of Ox- } \\
\text { ford, U.K. (pond } 1 \\
\text { in Ebert 1994a, } \\
1994 b \text { ), November } \\
1993\end{array}$ & $21.04 \% / 38 \%$ \\
\hline $\begin{array}{l}\text { Metschnikowiella biscuspidata } \\
\quad \text { (ascomycete fungi) }\end{array}$ & $\begin{array}{l}\text { Direct, horizontal; af- } \\
\text { ter the death of the } \\
\text { host; water- and } \\
\text { sediment-borne }\end{array}$ & $\begin{array}{l}\text { Infects hemolymph } \\
\text { (Metchnikoff 1884; } \\
\text { Green 1974) }\end{array}$ & $\begin{array}{l}\text { Pond south of Ox- } \\
\text { ford, U.K. (pond } 1 \\
\text { in Ebert } 1994 a, \\
1994 b \text { ), November } \\
1993\end{array}$ & $1.83 \% / 12 \%$ \\
\hline $\begin{array}{l}\text { Glugoides intestinalis } \\
\text { (formerly Pleistophora } \\
\text { intestinalis) } \\
\text { (Microsporidia) }\end{array}$ & $\begin{array}{l}\text { Direct, horizontal; } \\
\text { from the living and } \\
\text { dead host; } \\
\text { waterborne }\end{array}$ & $\begin{array}{l}\text { Infects epithelium of } \\
\text { the gut, causes diar- } \\
\text { rhea-like effect } \\
\text { (Ebert 1994a, } \\
\text { 1994b; Larsson et al } \\
\text { 1996) }\end{array}$ & $\begin{array}{l}\text { Pond south of Ox- } \\
\text { ford, U.K. (pond } 1 \\
\text { in Ebert } 1994 a, \\
1994 b \text { ), November } \\
1993\end{array}$ & Up to $100 \%^{b}$ \\
\hline $\begin{array}{l}\text { Ordospora colligata } \\
\quad \text { (formerly } P \text {. intestinalis) } \\
\quad \text { (Microsporidia) }\end{array}$ & $\begin{array}{l}\text { Direct, horizontal; } \\
\text { from the living and } \\
\text { dead host; } \\
\text { waterborne }\end{array}$ & $\begin{array}{l}\text { Infects epithelium of } \\
\text { the gut, causes diar- } \\
\text { rhea-like effect } \\
\text { (Larsson et al. } \\
\text { 1997) }\end{array}$ & $\begin{array}{l}\text { Pond } 50 \mathrm{~km} \text { north of } \\
\text { Moscow, Russia } \\
\text { (Mangin et al. } \\
\text { 1995), May } 1993\end{array}$ & $?^{\mathrm{b}}$ \\
\hline $\begin{array}{l}\text { Flabelliforma magnivora } \\
\text { (Microsporidia) }\end{array}$ & $\begin{array}{l}\text { Vertical through the } \\
\text { egg; most likely } \\
\text { horizontal transmis- } \\
\text { sion through a sec- } \\
\text { ond host }\end{array}$ & $\begin{array}{l}\text { Infects ovaries and fat } \\
\text { body (Mangin et al. } \\
\text { 1995; J. I. R. Lars- } \\
\text { son et al., } \\
\text { submitted) }\end{array}$ & $\begin{array}{l}\text { Pond } 50 \mathrm{~km} \text { north of } \\
\text { Moscow, Russia } \\
\text { (Mangin et al. } \\
\text { 1995), May 1993; } \\
\text { Pond } 20 \mathrm{~km} \text { north- } \\
\text { east of Oxford, } \\
\text { U.K., November } \\
1993\end{array}$ & $? / 50 \%$ \\
\hline
\end{tabular}

${ }^{a}$ Data (except those for Flabelliforma magnivora) are from Stirnadel and Ebert 1997. Data are for adult female Daphnia magna from three ponds in England that were sampled regularily over a 12 -mo period. The estimate for F. magnivora is from a single sample ( $n=22$ adult females) that was collected when these strains were collected.

${ }^{\mathrm{b}}$ The precise estimates for Glugoides intestinalis and Orodospora colligata are unknown because these two parasites are not easily distinguishable in the field. Unpublished data (D. Ebert) indicate that prevalences are up to 100\% (see Stirnadel 1994; Stirnadel and Ebert 1997).

cluding the controls) were tested for infections at the end of the experiment.

Population Experiments. These experiments were designed to quantify the effect of each parasite species or strain on small host populations. To do this, we started with infected or uninfected (control) populations of 10 hosts (ages 1-8 d) in glass jars filled with $300 \mathrm{~mL}$ of medium. We added food three times a week (producing a concentration of $2 \times 10^{6}$ cells algae/mL, independent of host density) and changed the medium once a week, during which hosts were counted. We did not transfer cadavers of dead hosts and other detritus to the new jars because transmission from a "spore bank" could influence the host-parasite dynamics (Ebert et al. 1997). The sole source of new infection was through reproduction of parasites within the cultures. The experiments were run for $16 \mathrm{wk}$ (experiment 3 only $15 \mathrm{wk}$ ), which represents about eight to 10 asexual host generations (see Perrin et al. 1992; Bengtsson 1995 for similar designs).

Since it was not possible to run all population experiments simultaneously, we ran the experiments in four groups (experiments 1-4). In experiment 1, we accidentally lost three (out of five) host population replicates in- 
fected with $P$. ramosa. We therefore included $P$. ramosa in experiment 2 as well.

Data Analysis. The design of the life table experiments allowed paired $t$-tests to be used for testing the effects of parasites on host fecundity and host age at death. For each pair of infected and uninfected D. magna, we calculated the relative fecundity as the cumulative (total) number of offspring produced by the infected host divided by the number of offspring produced by the control host, assuming the control host had died on the same day the infected host died. Thus, we did not calculate the relative lifetime reproductive effect. Instead, we calculated the relative fecundity over the same time period for the infected and uninfected females, which was until the parasite killed the infected host. The cumulative number of offspring was calculated disregarding the number of clutches a host produced (10 eggs from one clutch is equal to one egg from each of 10 clutches). This estimate of host relative fecundity is independent of a parasite's effect on host survival and corresponds to the variable $f$ in our model. It can range from 0 (instantaneous host castration) to 1 (no fecundity reduction).

In the population experiments, we recorded Daphnia density, Daphnia extinctions, and parasite presence. The time series from the infection treatments fell into three categories: first, host and parasite coexisted throughout the experiment; second, the parasite went extinct; and third the Daphnia and the parasites went extinct. Therefore, we did not treat all replicates as equal statistically. For example, if one of five replicate populations from an infection treatment lost the parasite, the subsequent rise in host population density in that replicate would raise the mean for all five replicates in a misleading way. We dealt with this statistical problem as follows: we excluded the first two time points from the time series because all experiments started with the same density. When parasite extinction occurred, we excluded the entire replicate because, in most of these cases, we could not determine when the parasite had gone extinct. Only for WBD were we able to track the continued presence of the infection because this bacterium makes its host bright white shortly after infection. Thus, for WBD we excluded the data only after the disease was seen for the last time in the culture. In cases where the hosts went extinct, we excluded the last two censuses before the last host had died. In those cases where neither host nor parasite went extinct, we had 14 (13 in experiment 3 ) data points per replicate. For the five parasite-free replicates, we calculated the mean density for each census day of the time series. These means were then used to calculate the relative density of each parasitized treatment (density of parasitized population/mean density of controls). For each of the replicates of the parasitized populations, we calculated the mean and the variance of these relative densities and the mean of the means and variances across all replicates to obtain an overall measure of relative host population density and of its variance. Variances were only calculated for populations in which neither host nor parasite had gone extinct so that only variances derived from an equal number of data points were being compared. For this reason, we did not calculate the variances of relative density for the $P$. ramosa and the WBD treatment.

This calculation of infected host population density relative to uninfected host population density has limitations in those cases where the parasite drove the host to extinction. This is particularly true for the WBD treatments, where either host or parasite had gone extinct in all replicates in $<10 \mathrm{wk}$. It was also the case for $P$. ramosa and M. biscuspidata. Other variations on how to calculate relative density yielded little difference from the results we report, so we believe that our estimates are reasonable. However, the relative density estimates for $P$. ramosa, $M$. biscuspidata, and WBD are probably overestimates because the last two density counts of those host populations that went extinct (perhaps with higher probability resulting from low densities) did not contribute to the calculations of relative density.

The relative densities were arcsine transformed and tested for deviation from 1 using a $t$-test. All analysis was done with the SAS statistics package (SAS Institute 1990).

\section{Results}

\section{Controls in Both Experiments}

All controls were verified to be uninfected at the end of the experiments by dissecting individuals and looking for signs of infection. Survival of control hosts was in all cases considerably better than survival of infected hosts (see below). Replicates of the control populations showed very similar patterns of change in population density. A typical pattern was a peak in Daphnia densities by $2-4$ wk followed by fluctuations around a mean density of $\sim 25-50$ animals. One unexplained extinction (out of a total of 20) was observed among the control replicates (experiment 3 ). Postmortem examination confirmed that this population was not parasitized.

\section{White Bacterial Disease}

WBD was the most virulent parasite used in this study. In the life table experiments, 18 of 20 animals became infected; of these, 15 died before maturity, and three produced one or two small clutches before dying (fig. 3; table 3 ; results of statistical tests for fecundity and survival of 


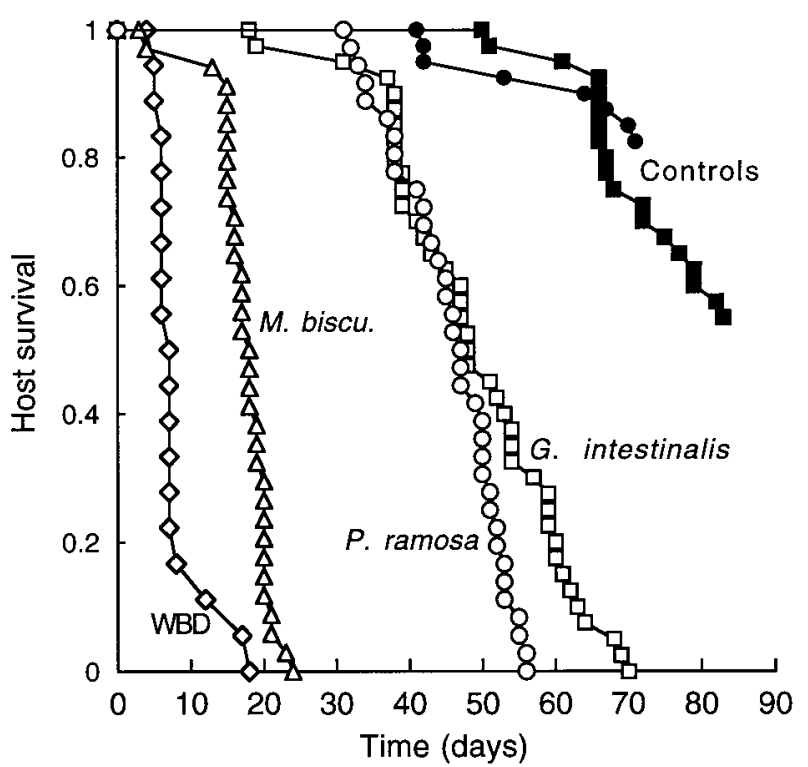

Figure 3: Survival curves of female Daphnia magna infected with four horizontally transmitted parasites (open symbols) and uninfected controls (filled symbols). Symbols: diamonds, white bacterial disease (WBD); triangles, Metschnikowiella biscuspidata; squares, Glugoides intestinalis; circles, Pasteuria ramosa. Since all controls for WBD and M. biscuspidata survived the death of all infected hosts, we do not show their survival curves.

infected and uninfected hosts are given in the tables). The two hosts that did not become infected survived until the end of the experiment and produced a total of 8 clutches and approximately as many eggs as the controls $(t=$ 0.63 , df $=20, P=.32$ ).

In the population experiments with $\mathrm{WBD}$, we recorded two cases of host extinction and the parasite went extinct in the other three cases (fig. 4). Overall reduction of host density was low (table 4) and difficult to evaluate because of the frequent parasite and host extinctions. The three replicate populations in which WBD went extinct grew slowly at first but caught up with the population density of the controls between weeks 6 and 9 (fig. 4). The instability of this host-parasite combination reflects well on our laboratory experience. White bacterial disease is difficult to maintain in our stock cultures.

\section{Pasteuria ramosa}

In the life table experiments, 36 of 40 hosts became infected. Mean age at death was $46 \mathrm{~d}$ and significantly shorter than survival of controls (fig. 3; table 3). Twenty-three of the 36 infected hosts never produced any eggs (table 3 ). Infected hosts that produced eggs never produced more than three clutches, while all controls produced $>11$ clutches before the end of the experiment. The four hosts that did not become infected survived until the end of the experiment and produced about the same number of eggs as did the control hosts $(t=1.54, \mathrm{df}=42, P=.13)$.

In the population experiments, Pasteuria ramosa drove all seven replicates of the host populations to extinction (fig. 4; table 4). The effect of this parasite on host extinctions was statistically significant compared to controls in both experiments (experiment 1: $P=.048, n=7$; experiment 2: $P=.004, n=10$; Fisher exact test). Extinction was typically preceded by a relatively long decline in density, much longer than the relatively rapid declines of populations driven to extinction by WBD and Metschnikowiella biscuspidata (see below). The long decline is a result of the relatively long life span of $P$. ramosa infected hosts.

\section{Metschnikowiella biscuspidata}

As with the two previous parasites, infectivity was high $(85 \%)$ in the life table experiments. Nineteen of the 34 infected hosts never produced eggs. The others produced very few eggs, mostly in a single clutch (table 3 ). The fecundity of the six hosts that did not become infected was $20.5 \%$ higher $(t=2.56, \mathrm{df}=41, P=.014)$ than that of the control animals, perhaps because of fecundity compensation by the hosts (sensu Minchella 1985). Infected hosts survived on average only $17.5 \mathrm{~d}$ (fig. 3; table 3).

In the population experiments, hosts went extinct in four of five replicates. Metschnikowiella biscuspidata went extinct in the fifth replicate (fig. 4; table 4). Compared to controls, infected host populations were significantly more likely to go extinct ( $P=.048, n=10$; Fisher exact test).

\section{Glugoides intestinalis}

This parasite is relatively benign (table 3 ), reducing host fecundity by $\sim 15 \%$, confirming the results of earlier studies (Ebert 1994b, 1995). Lethality of Glugoides intestinalis was comparable to $P$. ramosa (fig. 3), though fecundity reduction was much higher for $P$. ramosa (table 3). No host or parasite extinctions were observed in the population experiments (fig. 4; table 4). Glugoides intestinalis reduced host population density by $22 \%$ (table 4 ).

\section{Ordospora colligata}

Ordospora colligata and G. intestinalis are similar in pathology. Without ultrastructural analysis, they are morphologically nearly indistinguishable. Fecundity reduction by $O$. colligata was $\sim 20 \%$ (table 3 ). The mean age at host death was more than the 46-d length of the experiment (Mangin et al. 1995). Ordospora colligata did not reduce 
Table 3: Summary of results of life table experiments with five horizontally transmitted parasites

\begin{tabular}{|c|c|c|c|c|c|c|}
\hline Parasite & $\begin{array}{c}\text { Infectivity } \\
\text { (infected/ } \\
\text { sample size) }\end{array}$ & $\begin{array}{l}\text { Relative } \\
\text { fecundity } \\
( \pm \mathrm{SD})^{\mathrm{a}}\end{array}$ & $\begin{array}{l}\text { Proportion } \\
\text { of hosts } \\
\text { with no } \\
\text { offspring }\end{array}$ & $\begin{array}{c}\text { Mean age } \\
\text { at death } \\
(\mathrm{d} \pm \mathrm{SD})^{\mathrm{a}} / \\
\text { mortality rate } \\
(1 / \mathrm{d} \pm \mathrm{SD})\end{array}$ & $\begin{array}{l}\text { Time until } \\
\text { last } \\
\text { infected } \\
\text { host died/ } \\
\text { proportion } \\
\text { of dead } \\
\text { control at } \\
\text { this day }^{\mathrm{b}}\end{array}$ & $\begin{array}{l}\text { Duration } \\
\text { of } \\
\text { experiment } \\
(\mathrm{d})^{\mathrm{b}}\end{array}$ \\
\hline White bacterial disease & $90 \% 18 / 20$ & $.398 \pm .142^{* * *}$ & $83.3 \% 15 / 18$ & $7.94 \pm 3.80^{* * *} / .143 \pm .041$ & Day $18 / 0 \%$ & 35 \\
\hline $\begin{array}{l}\text { Pasteura ramosa } \\
\text { Metschnikowiella }\end{array}$ & $90 \% 36 / 40$ & $.037 \pm .057^{* * \star}$ & $63.9 \% 23 / 36$ & $45.97 \pm 6.98^{\star * *} / .022 \pm .004$ & Day $56 / 2.5 \%$ & 72 \\
\hline biscuspidata & $85 \% 34 / 40$ & $.131 \pm .224^{* * *}$ & $55.9 \% \quad 19 / 34$ & $17.53 \pm 3.49^{* * *} / .062 \pm .034$ & Day $24 / 0 \%$ & 35 \\
\hline Glugoides intestinalis & $100 \% 40 / 40$ & $.866 \pm .158^{\star * \star}$ & $0 \% 0 / 40$ & $49.47 \pm 11.42^{\star * *} / .021 \pm .007$ & Day $70 / 25 \%$ & 83 \\
\hline Ordospora colligata ${ }^{\mathrm{c}}$ & $100 \% 10 / 10$ & $.796^{\star}$ & $0 \% 0 / 10$ & $>46(50) /(.02)^{\mathrm{d}}$ & $\ldots$ & 46 \\
\hline
\end{tabular}

host population density or result in host or parasite extinctions in the experimental populations.

\section{Flabelliforma magnivora}

In all six strains of this microsporidium, host clutch size gradually decreased relative to the controls, and egg laying ceased a few days before host death. Relative fecundity across the six lines ranged from 0.87 to 0.32 (table 5). Relative fecundity and the total number of offspring produced differed significantly among the five strains isolated from the same natural population (table 6). Mean age to host death did not differ significantly among the isolates (table 6).

Flabelliforma magnivora drove its host populations extinct in two replicates of the population experiment (strains 2 and 9, fig. 5; table 5). The microsporidium went extinct in two replicates of strain 4 , indicating imperfect vertical transmission followed by the uninfected hosts rapidly outcompeting the infected hosts (fig. 5). Host extinction occurred in one of the five replicates of the Russian strain (fig. 5; table 5). In an earlier 12-wk experiment with host populations infected with this strain, no extinction occurred (Mangin et al. 1995).

\section{Comparison between Parasites and Strains}

In the following analysis, we treat all parasite species and the six strains of F. magnivora as independent data points. Therefore, these results suffer from a nonindependence of the data points. We address this problem in the discussion.

Relative fecundity and age at death of infected hosts appear to be only weakly correlated if at all (fig. 6). This is also true when vertically and horizontally transmitted parasites are considered separately.

Relative Fecundity. At the same relative fecundity levels, the vertically transmitted parasites reduced host density considerably more than did the horizontally transmitted parasites (fig. 7). Within each group, a positive trend is seen: the fewer offspring an infected host produces, the lower the density of an infected population. Using an ANCOVA, we found significant effects for mode of transmission (factor, $F=46.22, P<.0001$ ) and relative fecundity (covariable, $F=21.15, P=.002 ; r^{2}=0.88$, error mean squares $=0.0057$ ).

The occurrence of host extinctions in the population experiments was strongly correlated with relative host fecundity (Spearman's rank correlation: $\rho=-0.85, P=$ $.001, n=11$; fig. 8). At the same relative fecundity levels, the vertically transmitted parasites caused host extinction less often than did the horizontally transmitted parasites (ANCOVA: $r^{2}=0.85$, error mean squares $=0.0232$; mode of transmission [factor]: $F=6.46, P=.034$; relative fecundity [covariable]: $F=30.4, P=.0006)$.

Host Survival. Overall, a negative correlation was found between relative host density and age at death (Spearman's rank correlation: $\rho=-0.65, n=10, P=.04$; fig. 7). However, this correlation appears to be a consequence of the disjunct distribution of the two parasite groups (fig. 7). An ANCOVA with age at death as the covariable and mode of transmission as a factor did not reveal significant effects (mode of transmission [factor]: $F=3.53, P=$ 


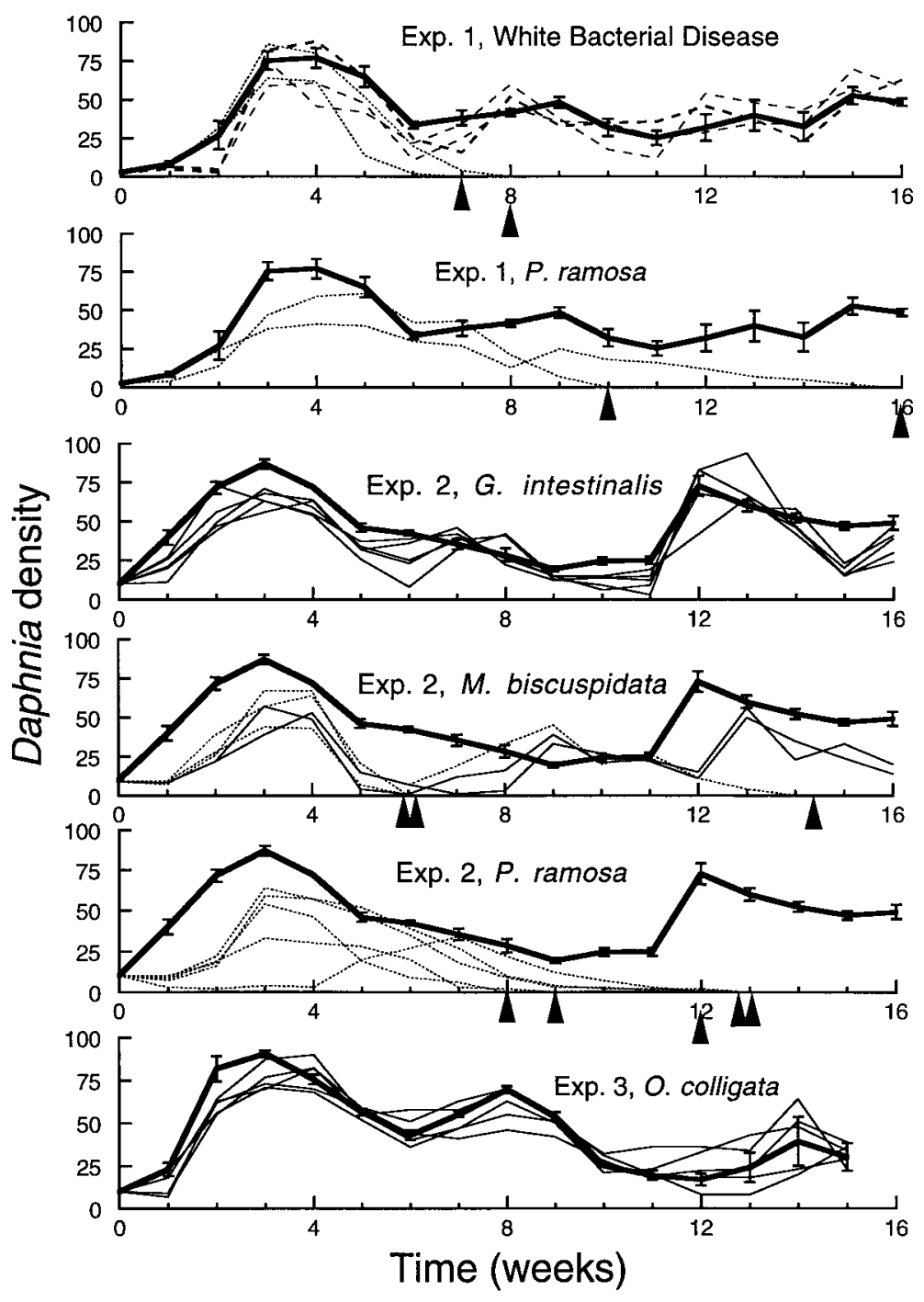

Figure 4: Population dynamics of uninfected Daphnia populations (thick line, means of five replicate populations \pm SE) and individual infected Daphnia populations (thin lines) from experiments 1,2 , and 3. Only horizontally transmitted parasites. The parasites were (from top to bottom): white bacterial disease, Pasteuria ramosa, Glugoides intestinalis, Metschnikowiella biscuspidata, P. ramosa, and Ordospora colligata. Infected populations are shown individually because they fall into three groups: host and parasite persisted throughout the study period (thin solid lines), host and parasite went extinct (dotted line), and the parasite went extinct, but host survived (stippled line). Host extinctions are indicated with black arrowheads. Within experiments, the same control populations were used.

.10 ; age at death [covariable]: $F=0.04, P=.85$, error mean squares $=0.0128$ ). No significant correlation was found between host extinction and age at death ( $\rho=-0.46, n=10, P>.1$; fig. 6 ). This does not change when a partial correlation was calculated with relative fecundity kept constant $(\rho=-0.15, n=10, P>.5)$.

Variance in Relative Density. Variances in relative density were more than seven times higher in the horizontally $(\bar{X} \pm$ SE: $0.25 \pm 0.15)$ than in the vertically $(0.033 \pm$
$0.0079)$ transmitted parasites ( $t$-test [log-transformed data]: $t=3.466, \mathrm{df}=7, P=.01)$. Within the three horizontally transmitted parasites for which it was possible to calculate the variance of relative density, the variance for M. biscuspidata was about three times higher than those for O. colligata and G. intestinalis (table 3). This goes hand in hand with the much higher host extinction rates in the M. biscuspidata treatments as compared to host extinction in the two microsporidian treatments.

Comparison between Models and Empirical Data. Figure 7 
Table 4: Summary of the results of population experiments with five horizontally transmitted parasites

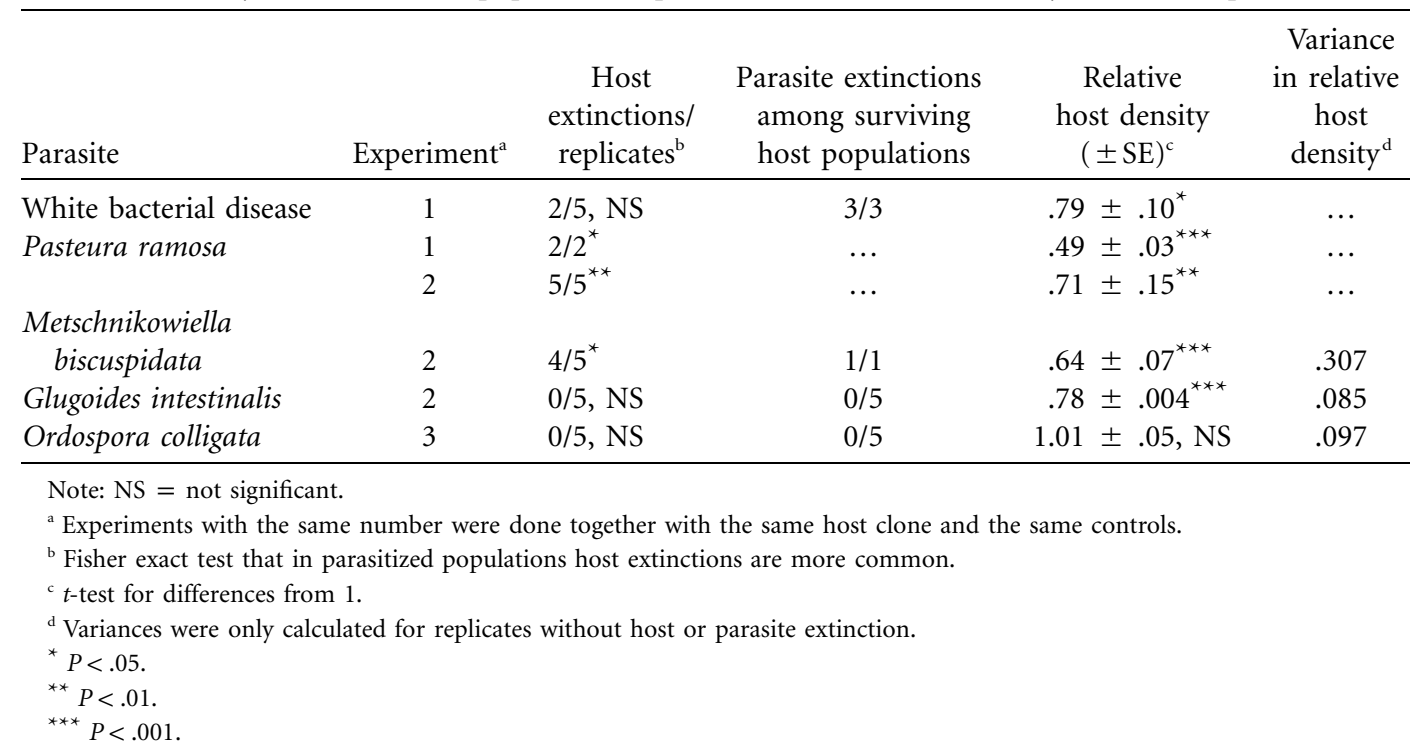

shows the predicted equilibrium host densities (horizontally transmitted parasites only) in relation to the relative fecundity and time to death, which were used as parameters in the deterministic model (model results are superimposed on the experimental data). The best fit is obtained for $O$. colligata, which, in agreement with the predictions of the model, did not cause a reduction in host density. In contrast G. intestinalis, which is as equally benign as O. colligata, reduced density by about $20 \%$, but the model predicts a reduction of $<2 \%$ for the simulated range of transmission rates. In agreement with the model, the three other horizontally transmitted parasites showed clear density reductions. The simulated equilibrium densities for a transmission rate of $\beta=0.01$ are closest to the observed data, but this might be misleading. Our estimates of relative density for these three parasites are likely to be overestimates (see "Material and Methods"). Because of the large number of extinctions in these three parasite treatments, our estimates of relative densities are also not as reliable as those obtained for the other parasites.

The results of the stochastic model indicate that host extinctions should be most frequent either when relative fecundity is very close to 0 or when relative fecundity and median time to host death are both low. This is in agreement with our results (cf. fig. 2 and table 4). As predicted for the two benign parasites, for O. colligata and G. intestinalis, no host extinctions were recorded. For the castrating parasite $P$. ramosa, which allows its host to survive for a long time (table 3 ), high extinction rates are predicted (fig. 2) and found (fig. 7; table 4). For WBD and $M$. biscuspidata, intermediate host extinction rates are predicted, with WBD-infected hosts having the lower observed and predicted extinction risks (fig. 2). Thus, for the given transmission rate, the rank orders in frequencies of predicted and observed host extinction show a good correspondence.

The stochastic model allowed us also to estimate parasite extinction rates. The only horizontal parasites that went extinct in some replicates (leaving the host population alive) were WBD and M. biscuspidata. Comparing figure $2 A$ and figure $2 B$ with each other shows that this is consistent with the results from the model. Further, in agreement with the model, no parasite extinctions are predicted and observed for the two benign microsporidian parasites. No conclusions can be drawn for extinctions of $P$. ramosa, as in all replicates the hosts went extinct.

As seen in figure 2, both host and parasite extinction rates depend on the transmission rate, $\beta$. A $\beta=0.1$ gives the best correspondence between predicted and observed extinction rates. While for benign parasites, such as $O$. colligata and G. intestinalis, no host or parasite extinctions are predicted within the huge range from $\beta=0.001-0.3$ (simulations not shown), for higher relative fecundities and median times to death, we find that decreasing $\beta$ leads to higher parasite extinction rates, while increasing $\beta$ leads to higher host extinction rates. Within the reasonable range of $\beta(0.01-0.1)$, the correspondence between the observed and predicted rank orders of extinction rates of the three more virulent horizontally transmitted parasites stays approximately the same (simulations not shown).

\section{Discussion}

The six parasite species used in this study differed strongly in their effects on host fecundity, host survival, host population density, and on the probability of driving their 
hosts or themselves to extinction. Parasite effects on individual hosts ranged from low to high lethality and from little impact on host fecundity to total sterilization. On the population level, one parasite had no effect on host density or population persistence, while others reduced host density strongly or even drove their hosts to extinction. This diversity in the outcomes of host-parasite interactions on a population level is predicted by epidemiological models and is in the center of our aims to understand the population dynamics of hosts and their parasites (Anderson and May 1978, 1979; May and Anderson 1978, 1979; Anderson 1979, 1982).

\section{Parasite-Induced Reduction in Host Density}

Our data reveal a tendency that the density of the host populations decline as host fecundity reduction increased (fig. 7). Even without knowledge of the transmission rates for our horizontally transmitted parasites, this trend is consistent with our deterministic epidemiological model (figs. 1, 7) as well as other similar models (Anderson 1979, 1982; Anderson and May 1981; McCallum 1994). For benign parasites, independent of their transmission rate, our model predicts no reduction in host population density. This prediction held true for one of the benign parasites in our study (Ordospora colligata), while the results for the other benign parasite, the microsporidum Glugoides intestinalis, went counter to the model's predictions and reduced host population density by $\sim 20 \%$. We have no explanation for this. Possibly the parasite was more harmful to hosts competing in the population experiments compared to its effect on isolated hosts in life table experiments.
Table 6: ANOVAs for testing differences between the five strains of Flabelliforma magnivora in the life table experiment and in the population experiment

\begin{tabular}{lcccc}
\hline Trait & Mean square & $\mathrm{df}$ & $F$ & $r^{2}$ \\
\hline $\begin{array}{l}\text { Life table experiments: } \\
\quad \text { Relative fecundity }\end{array}$ & .248 & $4 / 22$ & $11.49^{* * *}$ & .68 \\
$\quad$ Lifetime & & & & \\
$\quad$ reproduction & $2,660.7$ & $4 / 22$ & $16.42^{* * *}$ & .75 \\
$\quad$ Age at death & 212.5 & $4 / 22$ & $.99 \mathrm{NS}$ & .14 \\
$\begin{array}{l}\text { Population experiments: } \\
\quad \text { Relative host density }\end{array}$ & & & & \\
\hline
\end{tabular}

Note: NS $=$ not significant.

${ }^{* * *} P<.001$.

We found no relationship between the mean time to host death and relative host density in our population experiments. Horizontally transmitted parasites are predicted to suppress host density to the greatest degree when parasite pathogenicity levels are intermediate (Anderson 1979, 1982). Our model makes the same prediction; however, the simulations show that without increasing the mortality rate of infected hosts $(\alpha)$, this effect is hardly visible under a realistic range of parameters for relative fecundity, $f$, and mortality rate, $\alpha$, and parasite transmission rate, $\beta$ (fig. 1). The relationship between $\alpha$ and host equilibrium density depends strongly on $f$ and $\beta$. To empirically test how the average life span of infected hosts affects host population density would require a suite of parasite species or strains that exhibited a large range of effects on host mortality and a small or no range of effects on host fecundity. In contrast, the parasites in our experiment represented the opposite of this ideal; they had a large range of effects on the fecundities of infected hosts

Table 5: Summary of Flabelliforma magnivora experiments

\begin{tabular}{|c|c|c|c|c|c|c|}
\hline \multirow[b]{2}{*}{ Strain } & \multicolumn{2}{|c|}{ Life table experiments } & \multicolumn{4}{|c|}{ Population experiments } \\
\hline & $\begin{array}{l}\text { Relative } \\
\text { fecundity } \\
( \pm \mathrm{SD})^{\mathrm{a}}\end{array}$ & $\begin{array}{c}\text { Mean age } \\
\text { at death } \\
(\mathrm{d} \pm \mathrm{SD}) / \\
\text { mortality rate } \\
(1 / \mathrm{d} \pm \mathrm{SD})\end{array}$ & $\begin{array}{l}\text { Host } \\
\text { extinctions/ } \\
\text { replicates }\end{array}$ & $\begin{array}{c}\text { Parasite } \\
\text { extinction } \\
\text { among } \\
\text { surviving } \\
\text { host } \\
\text { populations }\end{array}$ & $\begin{array}{l}\text { Relative } \\
\text { host } \\
\text { density } \\
( \pm S E)^{a}\end{array}$ & $\begin{array}{c}\text { Variance } \\
\text { of relative } \\
\text { density }^{\mathrm{b}}\end{array}$ \\
\hline 2 & $.43 \pm .09^{* * *}$ & $57.75 \pm 11.1 / .018 \pm .004$ & $1 / 5$ & $0 / 4$ & $.35 \pm .02^{\star * \star}$ & .022 \\
\hline 4 & $.40 \pm .06^{* * *}$ & $49.00 \pm 1.4 / .020 \pm .001$ & $0 / 5$ & $2 / 5$ & $.43 \pm .04^{* * *}$ & .025 \\
\hline 5 & $.74 \pm .19^{\star *}$ & $67.0 \pm 15.77 / .016 \pm .005$ & $0 / 5$ & $0 / 5$ & $.59 \pm .02^{* * *}$ & .034 \\
\hline 8 & $.87 \pm .01^{* * *}$ & $48.00 \pm 2.83 / .021 \pm .001$ & $0 / 5$ & $0 / 5$ & $.64 \pm .02^{* * *}$ & .032 \\
\hline 9 & $.32 \pm .17^{* * *}$ & $57.33 \pm 18.04 / .022 \pm .017$ & $1 / 5$ & $0 / 4$ & $.41 \pm .03^{\star * *}$ & .018 \\
\hline Russia 1995 & $.55 \pm .11^{* * *}$ & $73.00 \pm 7.87 / .014 \pm .001$ & $1 / 5$ & $0 / 5$ & $.51 \pm .04^{* * *}$ & .061 \\
\hline
\end{tabular}

Note: Population data for strains 2, 4, 5, 8, and 9 were obtained in experiment 4 . The Russian strain was tested in experiment 3.

${ }^{a} t$-tests for differences between infected and controls.

${ }^{\mathrm{b}}$ Variances were only calculated for replicates without host or parasite extinction.

${ }^{* *} t>2.9, P<.01$

${ }^{* * *} t>3.8, P<.001$. 

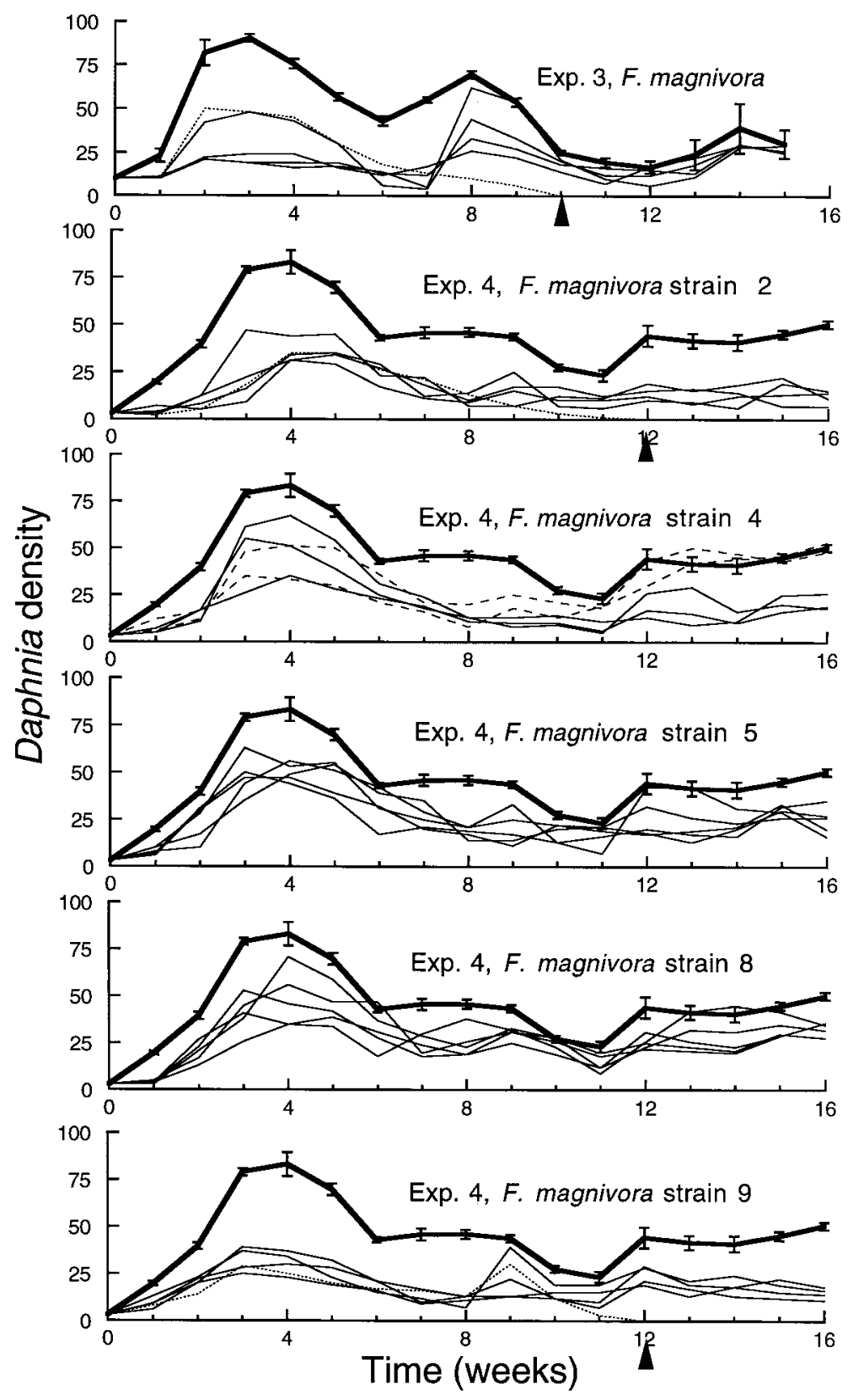

Figure 5: Population dynamics of uninfected Daphnia populations (thick line, means of five replicates \pm SE) and individual Flabelliforma magnivorainfected Daphnia populations (thin lines) from experiments 3 and 4. Infected populations are shown individually because they fall into three groups: host and parasite persisted throughout the study period (thin solid lines), host and parasite went extinct (dotted line), and parasite went extinct, but host survived (stippled line). Host extinctions are indicated with black arrowheads. Within experiments, the same control populations were used.

(from 0.037 to 0.86 , a 23.2 -fold difference between the most and least harmful to host fecundity), and a small range of effects on host mortality. Using the inverse of age at host death as an estimate of parasite-induced mortality rate, parasite-induced host mortality ranged from only $0.02-0.126$, a 6.3 -fold difference among the horizontally transmitted parasites. We are not aware of any Daphnia parasite that would kill infected hosts even earlier than WBD and thus would allow us to extend the range of $\alpha$.

The vertically transmitted parasites reduced host density significantly more than did the horizontally transmitted parasites. Because they were prevalent in all hosts at all 


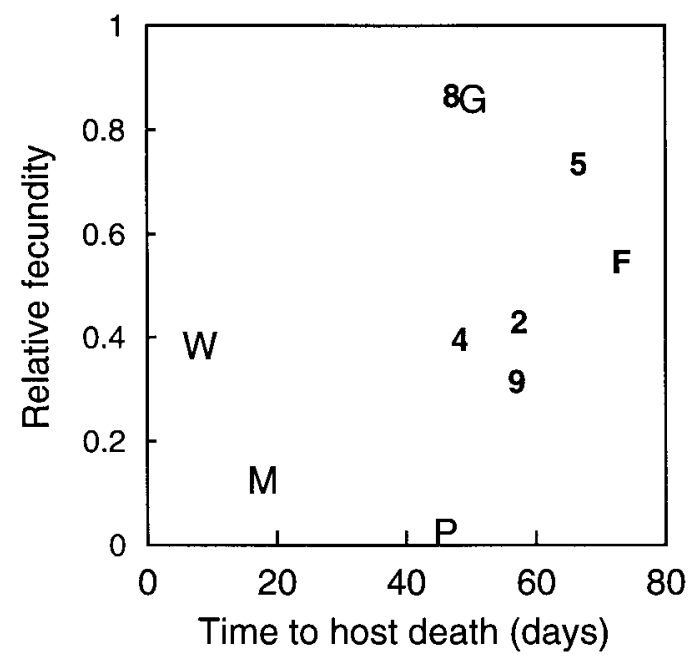

Figure 6: Relationship between relative fecundity and age of host at death for all parasite species and isolates used, except Ordospora colligata, for which no data for age at host death were available. The letters indicate the parasites: G, Glugoides intestinalis; M, Metschnikowiella biscuspidata; $P$, Pasteuria ramosa; $W$, white bacterial disease; $F$, Flabelliforma magnivora. The numbers indicate the five English strains of F. magnivora (cf. table 5). Thin-printed symbols indicate horizontal transmission, and bold symbols indicate vertical transmission.

times (100\% prevalence) in infected populations, they reduced the fecundity of every host in the population at all stages; while the hosts in cultures with horizontally transmitted parasites had some chance of reproducing normally before becoming infected, and their offspring each generation had the same chance. Furthermore, vertically transmitted parasites with $100 \%$ transmission may have a stronger effect on reducing host population densities than do horizontally transmitted parasites because vertical transmission is not reduced by low host density.

\section{Extinction of Hosts and Parasites}

Hosts and parasites were prone to extinction in certain experimental populations. Our stochastic model predicts that for very low relative fecundities and for the combinations of low relative fecundity and low host survival, host extinction rates may he high (fig. 2). This is caused by the combined effects of reduced host density and density oscillations. Our empirical data correspond with the predictions from the stochastic simulations quite well. While the two rather benign microsporidian parasites never caused host extinctions, the three more virulent horizontally transmitted parasites did so frequently. While, within a realistic range, the predictions for G. intestinalis and $O$. colligata do not depend on the transmission rate, the predictions for the three more virulent parasites do depend on transmission rates. For WBD, Pasteuria ramosa, and Metschnikowiella biscuspidata, an intermediate transmission rate of $\beta=0.1$ results in a good correspondence between predictions and data (cf. fig. 2 and table 4). However, extinction rates change with transmission rates and therefore the fit is less good for lower or higher transmission rates, although it does not change our conclusions.
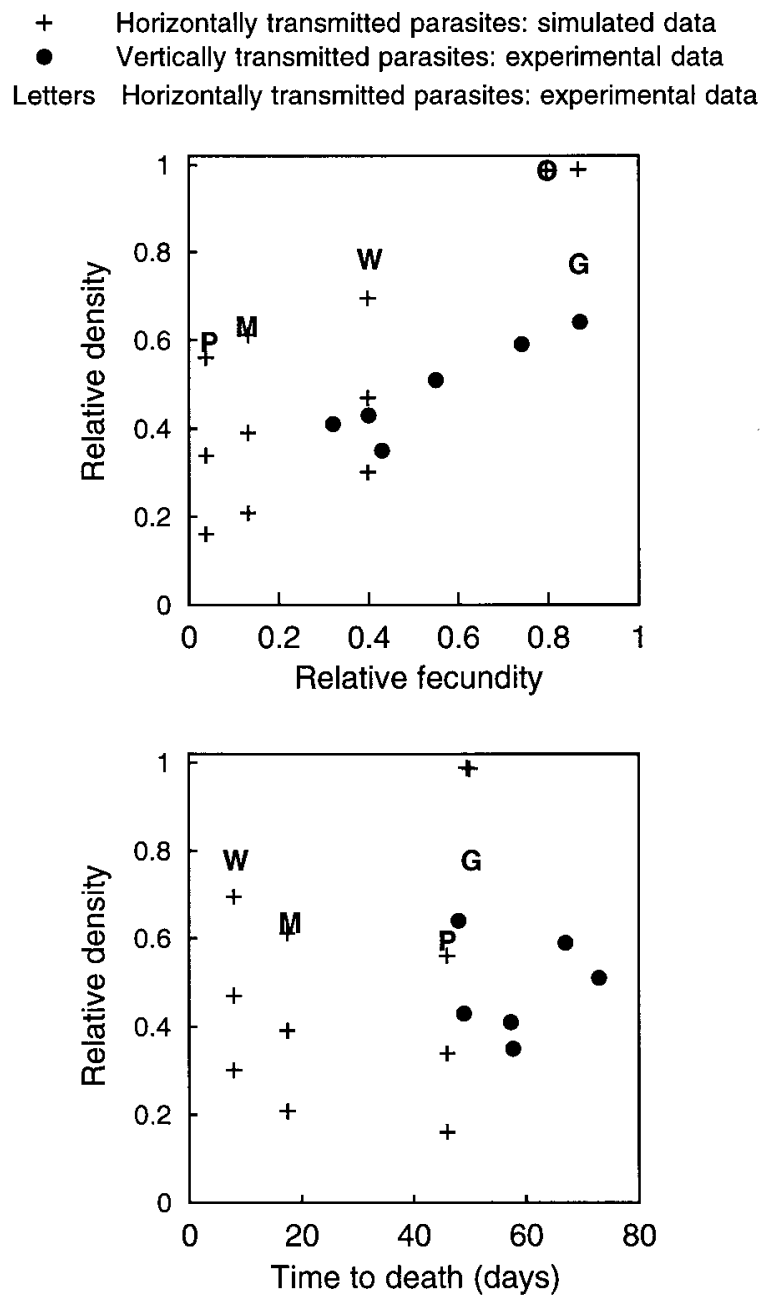

Figure 7: Relationship between the parasite's effect on the reduction in host density depending on relative fecundity and age at host death. The letters indicate the parasites: $G$, Glugoides intestinalis; $M$, Metschnikowiella biscuspidata; $O$, Ordospora colligata; $P$, Pasteuria ramosa; $W$, white bacterial disease; $F$, Flabelliforma magnivora. The dots indicate the six strains of $F$. magnivora (cf. table 5). The crosses show the relative density obtained from the model simulations for the horizontally transmitted parasites based on the parasite-specific parameter values (table 3). For each parasite, we show simulations with transmission rates of $\beta=0.01,0.03$, and 0.1 (the highest transmission rates resulted in the lowest relative densities). The relative densities for the simulated data for O. colligata and G. intestinalis are nearly 1.0 and overlap. Note that the lower graph does not show $O$. colligata because host survival data were not available. 

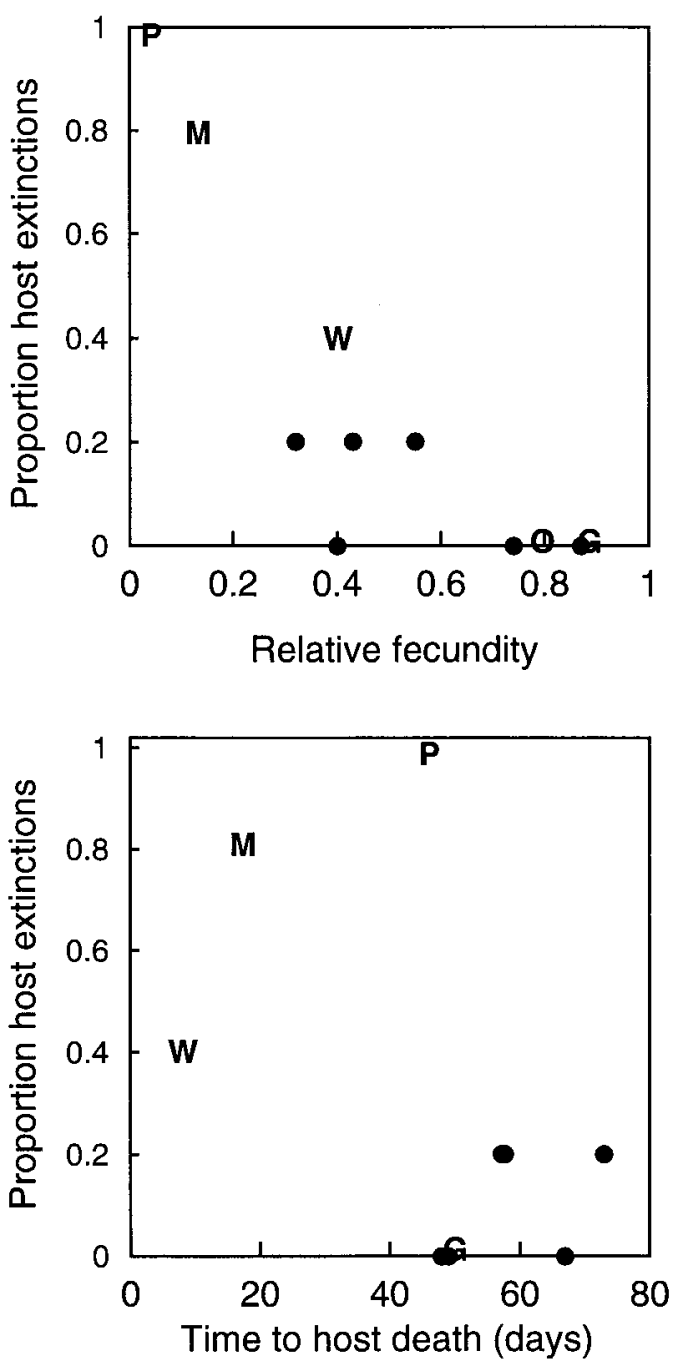

Figure 8: Relationship between the parasite's effect on the probability of host extinction depending on relative fecundity and age at host death. The letters indicate the parasites: G, Glugoides intestinalis; M, Metschnikowiella biscuspidata; $O$, Ordospora colligata; $P$, Pasteuria ramosa; $W$, white bacterial disease; $F$, Flabelliforma magnivora. The dots indicate the six strains of $F$. magnivora (cf. table 5). Note that the lower graph does not show $O$. colligata because host survival data were not available.

Given the same level of relative fecundity, the vertically transmitted parasites tended to drive their hosts less often to extinction than did the horizontally transmitted parasites (fig. 7). This may be explained by taking density oscillations into account. Despite their lower relative densities, the variance in density (a rough measure for the strength of oscillations) was significantly lower for the vertically transmitted parasite strains than the variance of the horizontally transmitted parasites. In fact, a simple model can show that for a realistic range of parameters oscillations are not expected for parasites that act like fixed del- eterious genes, as is the case for our vertically transmitted parasite strains (D. Ebert and M. Lipsitch, unpublished data).

Extinction of Daphnia magna because of parasitism has been suggested by Stirnadel (1994) in a natural population. She observed local extinctions of D. magna in a pond in South England after a period of strong parasitism with very frequent multiple infections.

As indicated by our stochastic results, parasites that drive their hosts extinct tend themselves to go extinct, even in cases when their hosts do not. White bacterial disease and M. biscuspidata either went extinct or drove their hosts extinct (table 4). Pasteuria ramosa went in all cases with its host extinct. Also consistent with the results from our simulation is that the benign $G$. intestinalis and O. colligata never went extinct. Virulent parasites were previously predicted and known to have high extinction rates, for example, virulent parasites cannot persist in small island populations (Anderson and May 1991).

\section{Parasite Prevalence}

Horizontally transmitted parasites that strongly reduce host survival and host fecundity are predicted to have low equilibrium prevalences of infection (Anderson and May 1991; McCallum 1994). Our experimental design did not allow us to quantify parasite prevalence without disrupting the experiment. However, prevalence estimates from field studies (table 1; Brunner 1996; Stirnadel and Ebert 1997; D. Ebert, unpublished data) show that the prevalences of the horizontally transmitted parasites match roughly the pattern predicted. The most prevalent parasites in nature were the ones that we found to be least harmful to their hosts. G. intestinalis and O. colligata had the lowest costs for their hosts and are generally found in high prevalences in the field (table 1; Brunner 1996; Stirnadel and Ebert 1997; D. Ebert, unpublished data). Pasteuria ramosa is similar in lethality to G. intestinalis but reduces host fecundity much more. Its prevalence in the field is much lower than those of G. intestinalis. The even more virulent WBD and M. biscuspidata are both found in low prevalences in nature.

\section{Consideration of Clade-Specific Effects}

As mentioned earlier, our comparison across parasites suffers from a lack of independence of the parasite species and isolates used. Some of the parasite species we used were phylogenetically distant (bacteria, fungus, and microsporidians). However, eight of the 11 isolates/species were microsporidians, with six of these being isolates of the same species. Thus, clade-specific effects might contribute to the results (Harvey and Pagel 1991), in particular 
to the comparisons between the effects of vertically versus horizontally transmitted parasites. However, parasiteinduced reductions in host survival and host fecundity are known to often show large amounts of genetic variance within parasite species (Davenport 1954; Fenner and Ratcliffe 1965; Parker 1985; Ebert 1994b) and rapidly react to selection (Fenner and Ratcliffe 1965; Bull et al. 1991; Ebert and Mangin 1997). Common ancestry and phylogenetic inertia are less important when the traits under investigation can evolve rapidly, that is, instantaneously on a paleontological timescale (Burt and Bell 1987). Nevertheless, to minimize confusion, we present our data in a manner that makes it possible to distinguish to what extent the analysis has been influenced by clade-specific effects.

\section{Conclusion}

Our study partially supports predictions about how effects of parasites on individual hosts are translated to population level effects. This is encouraging as the models on which our study is based are a major pillar of epidemiology in general. However, our study also highlights the difficulties in obtaining a good qualitative fit of the empirical data to the model. The transmission rates, which we were not able to assess for our parasites, are a very important part and necessary for a better understanding of the complete picture. Future tests of epidemiological models should take this into account.

Despite of the lack of transmission data, we were able to show that parasites with strong negative effects on host fecundity will reduce their host population densities and are more likely to cause host extinctions than are parasites with little effect on host fecundity. Extrapolating our results to more natural systems might be difficult, as natural populations are probably infected by more than one parasite species and as genetic variation for parasite virulence and host resistance is likely to be the rule rather than the exception in natural systems. That it is nevertheless possible to use simple models to predict dynamics in natural systems was shown by Hudson et al. (1998) for a helminth parasite infecting free-living grouse populations. Thus, even simple epidemiological models can be powerful tools in understanding the seemingly complex population dynamics of hosts and their natural enemies. This has implications for the use of parasites in biological control programs and for the role parasites play in driving small populations to extinction (Anderson 1982; McCallum and Dobson 1995).

\section{Acknowledgments}

We thank S. Hodge, R. Jones, D. McKee, and J.W. Wilde for help during various stages of the experimental work.
B. Grenfell, A. Herre, H. McCallum, W. Weisser, J.W. Wilde, and one anonymous reviewer greatly improved the manuscript with their comments. This work was supported by the North Atlantic Treaty Organization and the National Science Foundation, U.S.A. (K.L.M.), the Natural Environment Research Council, U.K. (K.L.M and D.E.), and the Swiss Nationalfond (D.E.).

\section{Literature Cited}

Anderson, R. M. 1979. Parasite pathogenicity and the depression of host population equilibria. Nature (London) 279:150-152.

- 1982. Theoretical basis for the use of pathogens as biological control agents of pest species. Parasitology 84:3-33.

Anderson, R. M., and R. M. May. 1978. Regulation and stability of host-parasite population interactions. I. Regulatory processes. Journal of Animal Ecology 47: 219-247.

- 1979. Population biology of infectious diseases. I. Nature (London) 280:361-367.

- 1981. The population dynamics of microparasites and their invertebrate hosts. Philosophical Transactions of the Royal Society London B, Biological Sciences 291: 451-524.

1986. The invasion, persistence and spread of infectious disease within animal and plant communities. Philosophical Transactions of the Royal Society of London B, Biological Sciences 314:533-570.

. 1991. Infectious diseases of humans. Oxford University Press, Oxford.

Bengtsson, J., and G. Milbrink. 1995. Predicting extinctions: interspecific competition, predation and population variability in experimental Daphnia populations. Oecologia (Berlin) 101:397-406.

Brunner, D. U. 1996. The role of population size, migration, parasites and competition for the genetic population structure of Daphnia magna. Diploma thesis, University of Basel, Basel.

Bull, J. J., I. J. Molineux, and W. R. Rice. 1991. Selection of benevolence in a host-parasite system. Evolution 45: 875-882.

Burt, A., and G. Bell. 1987. Red Queen versus Tangles Bank models. Nature (London) 330:118.

Davenport, F. M. 1954. The inequality of potential in influenza virus for adaptation to mice. Journal of Immunology 72:485-488.

Dobson, A. P., and P. J. Hudson. 1986. Parasites disease and the structure of ecological communities. Trends in Ecology \& Evolution 1:11-15.

Ebert, D. 1994a. Genetic differences in the interactions of 
a microsporidian parasite and four clones of its cyclically parthenogenetic host. Parasitology 108:11-16.

. 1994b. Virulence and local adaptation of a horizontally transmitted parasite. Science (Washington, D.C.) $265: 1084-1086$.

- 1995. The ecological interactions between a microsporidian parasite and its host Daphnia magna. Journal of Animal Ecology 64:361-369.

Ebert, D., and K. L. Mangin. 1997. The influence of host demography on the evolution of virulence of a microsporidian gut parasite. Evolution 51:1828-1837.

Ebert, D., P. Rainey, T. M. Embley, and D. Scholz. 1996. Development, life cycle, ultrastructure and phylogenetic position of Pasteuria ramosa Metchnikoff 1888: rediscovery of an obligate endoparasite of Daphnia magna Straus. Philosophical Transactions of the Royal Society of London B, Biological Sciences 351:1689-1701.

Ebert, D., R. J. H. Payne, and W. W. Weisser. 1997. The epidemiology of parasitic diseases in Daphnia. Pages 91-111 in K. Dettner, G. Bauer, and W. Völkl, eds. Vertical food web interactions: evolutionary patterns and driving forces. Springer, Heidelberg.

Fenner, F., and F. N. Ratcliffe. 1965. Myxomatosis. Cambridge University Press, Cambridge.

Fine, P. E. M. 1975. Vectors and vertical transmission: an epidemiologic perspective. Annals of the New York Academy of Science 266:173-194.

Finlayson, L. H. 1949. Mortality of Laemophloeus (Coleoptera, Cucujidae) infected with Mattesia dispora Naville (Protozoa, Schizogregarinaria). Parasitology 40: 261-264.

Green, J. 1974. Parasites and epibionts of Cladocera. Transactions of the Zoological Society of London 32:417-515.

Harvey, P. H., and M. D. Pagel. 1991. The comparative method in evolutionary biology. Oxford University Press, Oxford.

Herre, E. A. 1993. Population structure and the evolution of virulence in nematode parasites of fig wasps. Science (Washington, D.C.) 259:1442-1445.

Hudson, P. J., D. Newborn and A. P. Dobson. 1992. Regulation and stability of a free-living host-parasite system: Trichostrongylus tenuis in red grouse. I. Monitoring and parasite reduction experiments. Journal of Animal Ecology 61:477-486.

Hudson, P. J., A. P. Dobson, and D. Newborn. 1998. Prevention of population cycles by parasite removal. Science (Washington, D.C.) 282:2256-2258.

Keymer, A. E. 1981. Population dynamics of Hymenolepis diminuta in the intermediate host. Journal of Animal Ecology 50:941-950.

Klein, J., and M. Moeschberger. 1997. Survival analysis: techniques for censored and truncated data. Springer, New York.
Klüttgen, B., U. Dülmer, M. Engels, and H. T. Ratte. 1994. $\mathrm{ADaM}$, an artificial freshwater for the culture of zooplankton. Water Research 28:743-746.

Kohler, S. L., and M. J. Wiley. 1992. Parasite-induced collapse of populations of a dominant grazer in Michigan streams. Oikos 65:443-449.

Larsson, J. I. R., D. Ebert, J. Vavra, and V. N. Voronin. 1996. Redescription of Pleistophora intestinalis Chatton, 1907, a microsporidian parasite of Daphnia magna and Daphnia pulex, with establishment of the genus Glugoides (Microspora, Glugeidae). European Journal of Protistology 32:251-261.

Larsson, J. I. R., D. Ebert and J. Vavra. 1997. Ultrastructural study of Ordospora colligata gen. et sp. nov. (Microspora, Ordosporidae Fam. Nov.), a new microsporidian parasite of Daphnia magna (Crustacea, Cladocera). European Journal of Protozoology 33:432-443.

Larsson, J. I. R., D. Ebert, K. L. Mangin, and J. Vavra. 1998. Ultrastructural study and description of Flabelliforma magnivora n. sp. (Microspora, Duboscqiidae), a microsporidian parasite of Daphnia magna (Crustacea, Cladocera, Daphniidae). Acta Protozoologica 37:41-52.

Lipsitch, M., M. A. Nowak, D. Ebert, and R. M. May. 1995. The population dynamics of vertically and horizontally transmitted parasites. Proceedings of the Royal Society of London B, Biological Sciences 260:321-327.

Mangin, K. L., M. Lipsitch, and D. Ebert. 1995. Virulence and transmission modes of two microsporidia in Daphnia magna. Parasitology 111:133-142.

May, R. M. 1974. Stability and complexity in model ecosystems. Princeton University Press, Princeton, N.J.

May, R. M., and R. M. Anderson. 1978. Regulation and stability of host-parasite population interactions. II. Destabilizing processes. Journal of Animal Ecology 47: 249-268.

- 1979. Population biology of infectious diseases. II. Nature (London) 280:455-461.

- 1983. Epidemiology and genetics in the coevolution of parasites and hosts. Proceedings of the Royal Society of London B, Biological Sciences 219:281-313.

McCallum, H. 1994. Quantifying the impact of disease on threatened species. Pacific Conservation Biology 1: 107-117.

- 1995. Modelling wildlife-parasite interactions to help plan and interpret field studies. Wildlife Research 22:21-29.

McCallum, H., and A. Dobson. 1995. Detecting disease and parasite threats to endangered species and ecosystems. Trends in Ecology \& Evolution 10:190-194.

Metchnikoff, M. E. 1884. Über eine Sprosspilzkrankheit der Daphniden. Beitrag zur Lehre der Phagocyten gegen Krankheitserreger. Virchows Archiv für pathologische Anatomie und Physiologie 9:177-193. 
1888. Pasteuria ramosa un représentant des bactéries a division longitudinale. Annales de L'Institut Pasteur (Paris) 2:165-170.

Minchella, D. J. 1985. Host life-history variation in response to parasitism. Parasitology 90:205-216.

Minchella, D. J., and M. E. Scott. 1991. Parasitism: a cryptic determinant of animal community structure. Trends in Ecology \& Evolution 6:250-254.

Park, T. 1948. Experimental studies on interspecies competition. I. Competition between populations of the flour beetles Trifolium confusum (Duval) and Tribolium cansteneum (Herbst). Ecological Monographs 18: 265-307.

Parker, M. A. 1985. Local population differentiation for compatibility in an annual legume and its host-specific fungal pathogen. Evolution 39:713-723.

Perrin, N., D. J. Baird, and P. Calow. 1992. Resource allocation, population dynamics and fitness: some experiments with Daphnia magna Straus. Archiv für Hydrobiologie 123:431-449.
Roelke-Parker, M. E., L. Munson, C. Packer, R. Kock, S. Cleaveland, M. Carpenter, S. J. O'Brien, et al. 1996. A canine distemper virus epidemic in Serengeti lions (Panthera leo). Nature (London) 379:441-445.

SAS Institute. 1990. SAS/STAT user's guide, release 6.06 ed. SAS Institute, Cary, N.C.

Scott, M. E., and A. Dobson. 1989. The role of parasites in regulating host abundance. Parasitology Today 5: 176-183.

Stirnadel, H. A. 1994. The ecology of three Daphnia species - their microparasites and epibionts. Diploma thesis, University of Basel, Basel.

Stirnadel, H. A., and D. Ebert. 1997. Prevalence, host specificity and impact on host fecundity of microparasites and epibionts in three sympatric Daphnia species. Journal of Animal Ecology 66:212-222.

Associate Editor: Bryan T. Grenfell 